\title{
Detection and Enhancement of Small Masses via Precision Multiscale Analysis
}

\author{
Dongwei Chen ${ }^{1}$, Chun-Ming Chang ${ }^{1}$, and Andrew Laine ${ }^{2}$ \\ 1 Electrical and Computer Engineering, University of Florida, Gainesville, FL 32611 \\ 2 Center for Biomedical Engineering, Columbia University, New York, NY 10027
}

\begin{abstract}
We introduce a continuous scale wavelet detector. Our algorithm was able to detect a mass that could not be seen using conventional windowing and leveling or traditional methods of contrast enhancement. An artifact free enhancement algorithm based on overcomplete multiscale wavelet analysis is then presented. The novelty of this algorithm lies in its detection of directional features and removal of unwanted perturbations.
\end{abstract}

\section{Introduction}

Mammography is the most effective tool for the early detection of breast cancer. However, it has been suggested that mammograms as normally viewed, display only about $3 \%$ of the total information detected.

Wavelet algorithms have found widespread use in detection and enhancement applications, i.e., multiscale representations based on wavelets have been previously carried out for mammographic feature analysis[3]. The local nature of time by wavelet analysis enables capturing the beginning of transients and provides transient representation by a small number of coefficients. Such redundant wavelet representations provide the property of shift invariance which is useful for image analysis tasks.

In this paper, we first use a continuous wavelet detector to locate suspicious regions. An enhancement algorithm based on overcomplete multiscale wavelet analysis is then applied. The novelty and advantage of this algorithm compared to existing techniques lies in its detection of directional features and suppression of local perturbations (artifacts).

\section{Continuous Scale Discrete Wavelet Detector}

Let's first consider the hypotheses $H_{1}: y(x)=s(x)+n(x)$ and $H_{2}: y(x)=n(x)$, where $x$ is a location, $y$ is an observed signal, $s$ is an underlying signal to be detected, and $n$ is additive noise. Based on an observation $y$, a wavelet detection algorithm is used to choose between $H_{0}$ and $H_{1}$ by comparing the detector's output with a threshold determined by a statistical measure[5]. In particular, we shall develop a continuous scale wavelet detector compared to a M-Band wavelet detector[5]. 


\subsection{A Continuous Scale Discrete Wavelet Transform}

The Continuous Scale Discrete Wavelet Transform (CSDWT) is defined as the CWT sampled along the shifting parameter,

$$
\mathbf{W} f(a, n)=\int_{-\infty}^{\infty} f(t) \psi\left(\frac{n-t}{a}\right) d t
$$

Scaling Space First, let's introduce scaling space to describe signals of distinct resolution. A compactly supported scaling function $\phi(t)$ satisfies the following conditions [10] :

1. Riesz basis condition: $A \leq \sum_{k \in \mathcal{Z}}|\Phi(\omega+2 k \pi)|^{2} \leq B$, where A and B are strictly positive constants;

2. Order property: $\Phi(0)=1, \Phi^{(m)}(2 k \pi)=0, k \in \mathcal{Z}, k \neq 0$, for $m=0, \ldots, N-1$;

3. Two-scale relation: $\phi\left(\frac{t}{2}\right)=\sum_{k \in \mathcal{Z}} h(k) \phi(t-k)$;

4. Frequency constraint: $\Phi(\omega) \neq 0$, for $|\omega| \leq \pi$, and $\Phi(\omega)$ should be very small for $|\omega|>\pi$.

Initial Condition In the traditional DWT analysis literature, we assume that $x(n)=\int_{-\infty}^{\infty} f(t) \phi(n-t) d t$. However, in most cases, this assumption is not true. When the impulse response of an acquisition device is an ideal lowpass filter $\xi(t)$

$x(n)=\int_{-\infty}^{\infty} f(t) \xi(n-t) d t$. If the scaling function is a spline $\beta^{n}(t)$ of order $n-1$, $\xi(t) \approx \sum_{k}\left(b^{n}\right)^{-1}(k) \beta^{n}(t-k), x^{\prime}(n)=\sum_{k} x(k)\left(b^{n}\right)^{-1}(n-k)=\int_{-\infty}^{\infty} f(t) \phi(n-t) d t$. Therefore, we need to initialize data $x(n)$ with a prefilter $\left(b^{n}\right)^{-1}(k)$.

CSDWT A discrete approximation of $f(t)$ at scale $a$ is defined as

$$
\mathbf{S}_{a}^{d} f(n)=\int_{-\infty}^{\infty} f(t) \phi\left(\frac{n-t}{a}\right) d t
$$

It can be shown from the two-scale relation and $\mathrm{Eq}(2)$ that

$$
\mathcal{F}\left\{\mathbf{S}_{2^{j}}^{d}+1 f(n)\right\}=H\left(2^{j} \omega\right) \mathcal{F}\left\{\mathbf{S}_{2^{j}}^{d} f(n)\right\} .
$$

From the initial condition, we have $\mathbf{S}_{1} f(n)=x^{\prime}(n)$, where $a=1$ is the finest scale of analysis. As a increases, $\mathbf{S}_{a} f(n)$ becomes a coarser representation of $f(t)$.

Theorem 1. Given $x(n)$, for $a>s_{0}$, if $\tilde{\Psi}_{a}(\omega)$ is band-limited between $[-\pi, \pi]$, the CSDWT can be calculated by $\mathbf{W}_{a} f(a, n)=\sum_{k \in \mathcal{Z}} p(a, k) x(n-k)$, where $P^{c}(a, \omega)=\frac{\tilde{\Psi}_{a}(\omega)}{\Phi(\omega)}, P^{c}(a, \omega)=\int_{-\infty}^{\infty} p^{c}(a, t) e^{-j \omega f_{s} t} d t, \quad p(a, k)=\left.p^{c}(a, t)\right|_{t=k}$. 
Proof. Note that $\tilde{\psi}_{a}(t)$ and $p^{c}(a, t)$ are band-limited at $[-\pi, \pi]$. We see that

$$
\tilde{\psi}_{a}(t)=\int_{-\infty}^{\infty} p^{c}(a, u) \phi(t-u) d u .
$$

If we substitute $\mathrm{Eq}(4)$ into $\mathrm{Eq}(1)$ and exchange the sequency of the integral, we obtain

$$
\mathbf{W}_{a} f(a, n)=\int_{-\infty}^{\infty} x^{c}(n-u) p^{c}(a, u) d u .
$$

It is straightforward to show that if $x^{c}(t)$ and $p^{c}(a, t)$ are band-limited to $[-\pi, \pi]$, $\int_{-\infty}^{\infty} x^{c}(n-u) p^{c}(a, u) d u=\sum_{k \in \mathcal{Z}} p(a, k) x(n-k)$. Therefore,

$$
\mathbf{W}_{a} f(a, n)=\sum_{k \in \mathcal{Z}} p(a, k) x(n-k) .
$$

If a wavelet $\psi(t)$ is local in the frequency domain, we need to select an $s_{0}$ such that $\tilde{\Psi}_{s 0}(\omega)=\Psi_{s 0}(\omega) \operatorname{Rect}(\omega)$ has an acceptable approximation error onto the scaling space. The approximation error is defined as the ratio of the energy of $\Psi(a \omega)$ outside $[-\pi, \pi]$ to the total energy and decreases as $s_{0}$ increases. It is straightforward to show that $\tilde{\psi}_{s 0}(t)$ is also a mother wavelet.

When little is known about the best scale to detect a certain feature, it is desirable to first search the scale dyadically, then carry out a finer search. When an $s_{0}$ is selected, it may be considered as a "voice" and $P\left(2^{L} s_{0}, \omega\right)$ can be calculated as $[9]$

$$
P\left(2^{L} s_{0}, \omega\right)=P\left(s_{0}, 2^{L} \omega\right)\left(\prod_{k=0}^{L-1} H\left(2^{k} \omega\right)\right) .
$$

Thus, $\mathrm{Eq}(3),(6)$ and (7) yield

$$
\mathcal{F}\left\{\mathbf{W}_{2^{j} s 0} f\right\}=P\left(s_{0}, 2^{j} \omega\right) \mathcal{F}\left\{\mathbf{S}_{2^{j}} f\right\} .
$$

Let $K(\omega)$ be a $2 \pi$ periodic function and suppose $k(\omega)$ satisfies

$$
K(\omega) P\left(s_{0}, \omega\right)+|H(\omega)|^{2}=1
$$

It is straightforward to derive from $\mathrm{Eq}(9),(3)$ and (8) that

$$
\mathcal{F}\left\{\mathbf{S}_{2^{j}} f\right\}=H^{*}\left(2^{j} \omega\right) \mathcal{F}\left\{\mathbf{S}_{2^{j}}+1 f\right\}+K\left(2^{j} \omega\right) \mathcal{F}\left\{\mathbf{W}_{2^{j}} f\right\} .
$$

Thus, $\mathrm{Eq}(3),(8)$ and (10) show that a signal can be decomposed and reconstructed at an arbitrary scale $2^{J-1} s_{0}$.

\subsection{Application of CSDWT detector}

In this study, the wavelet applied was a second derivative of a spline of order 5 $(\mathrm{n}=4, \mathrm{~d}=2)$. The scaling function and mother wavelet is given by

$$
\Phi(\omega)=\left(\frac{\sin \left(\frac{\omega}{2}\right)}{\frac{\omega}{2}}\right)^{n}, \quad \Psi(\omega)=(j \omega)^{d}\left(\frac{\sin \left(\frac{\omega}{4}\right)}{\frac{\omega}{4}}\right)^{n+d}
$$


and

$$
H(\omega)=e^{j t 0 \omega} \cos ^{n}\left(\frac{\omega}{2}\right), \quad P\left(s_{0}, \omega\right)=e^{j t 1 \omega} \frac{j^{d} 2^{n+2 d}}{s_{0}^{n}} \frac{\sin ^{n+d}\left(\frac{s 0 \omega}{4}\right)}{\sin ^{n}\left(\frac{\omega}{2}\right)} .
$$

$t_{0}=\frac{1}{2}$ when $n$ is odd and level $L=0$. Otherwise, $t_{0}=0 . t_{1}=\frac{1}{2}$ when $d$ is odd and level $L=0, t_{1}=0$.

We first applied our detector to radiographic images of an RMI phantom, captured with technique factors of $22 \mathrm{kVp}$ and $112 \mathrm{mAs}$. The smallest mass in the insert of the phantom cannot be seen using conventional window and leveling at 12-bit contrast resolution. In Figure 1(b), all masses within the phantom were detected correctly. Note that the scale used was not dyadic. Detectors searching at dyadic scales alone would miss the smallest mass, located in the upper right corner.

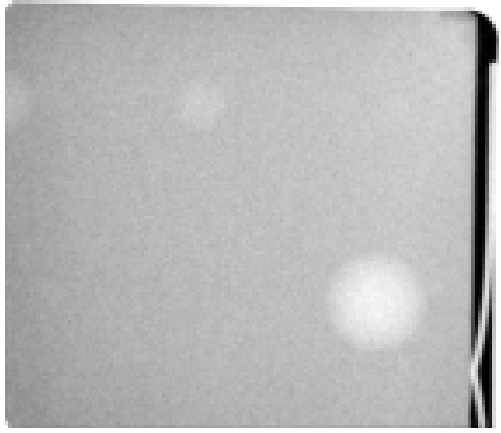

(a)

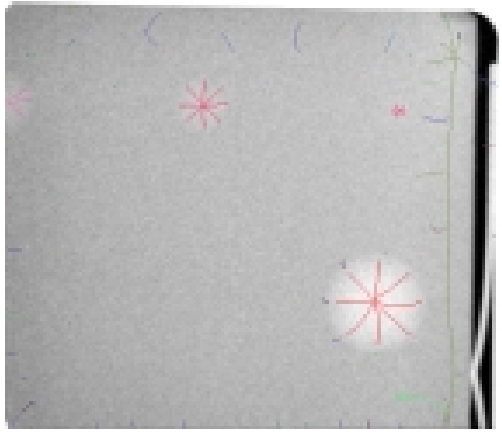

(b)

Fig. 1. (a) Digital X-ray image of the RMI156 phantom. (b) Maxima from precision scale detector obtained at scale 53.8 .

We applied our detector to 6 cases of real mammograms with dense tissue. All the masses were detected correctly. Figure 2(b) shows a sample result.

\section{Enhancement of Mammograms from Oriented Information}

Reliable diagnosis by radiographs of malignant breast disease depends on observing local and distant changes in tissues produced by the disease. Of the visual signs of cancer found by radiologists, spiculated features are of great importance. Unfortunately, at the early stages of breast cancer, these signs are very subtle and varied in appearance, making diagnosis difficult and challenging even to specialists in mammography. In this section, an enhancement algorithm based on multiscale wavelet analysis is described. The novelty and advantage of this algorithm lies in its detection of spiculated features and removal of unwanted pertabations (artifacts). 


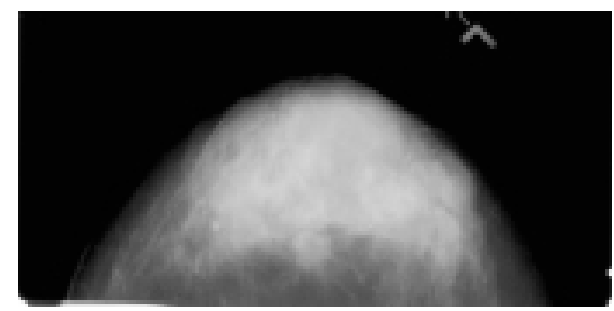

(a)

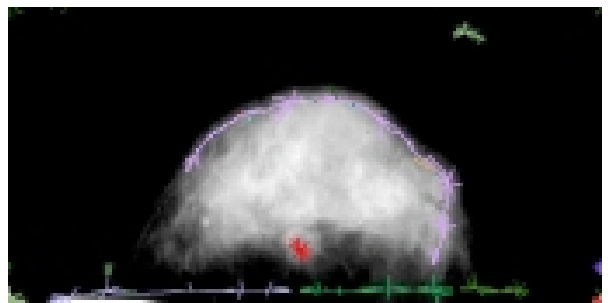

(b)

Fig. 2. (a) Mammogram lcc004. (b) Precision maxima superimposed on lcc004 enhanced by window and leveling.

\subsection{Measure of Coherence}

Texture plays an important role in many machine vision and image processing tasks including surface inspection, scene classification, surface orientation and shape determination. Texture patterns may be characterized by extracting measurements that quantify the nature and directions of pattern. Most breast carcinomas have the appearance of stellate lesions consisting of a central mass surrounded by radiating spicules. The spicules radiate outward in all directions and vary in length. These provide an important cue for early detection of breast cancer.

Rao and Schunck [8] defined the orientation field of a texture image to consist of two images - an angle image and a coherence image. The angle image denotes the dominant local orientation at each point and the coherence image represents the degree of anisotropy at each point. They strongly advocated the use of angle and coherence images as intrinsic images. In this paper, we investigated the efficiency of these two representations to capture and enhance features of importance to mammography.

\subsection{Methodology}

Our algorithm consists of the following four steps.

(1) Multiscale Wavelet Analysis: Wavelet transforms, owing to their localization characteristics, are powerful tools of analysis for many signal and image processing applications. Through multiscale analysis we can extract features at distinct scales and provide local information often hidden in an original (single resolution) mammogram. One major drawback of traditional wavelet transforms is their lack of translation invariance, making the content of wavelet subbands unstable under the translations of an input signal. In our algorithm, a digitized mammogram was decomposed using a fast wavelet transform algorithm (FWT) [4]. In order to obtain wavelet coefficients at each level without downsampling, a undecimated "algorithme à trous" (algorithm with holes) [2] was implemented. In the spatial domain, this redundancy corresponds to a representation without aliasing. 
(2) Separable Steerable Filters: A filter is called "steerable" if the filter at an arbitrary orientation can be expressed as a linear combination of a set of basis filters, generated from rotations of a single kernel [1]. Steerable filters [1], which can be adaptively adjusted to arbitrary orientation, are used to detect stellate patterns of spicules and locate feature orientations more precisely. As pointed out by [7], the separability property of the filters speeds up computations considerably when convolved with large image matrices. In our algorithm, we used three basis functions as steerable filters. The $x-y$ separable steerable approximations of filter kernels were generated by Singular Value Decomposition (SVD) [1, 7]. Using a set of separable steerable filters, the magnitude $\left(M^{i}\right)$ and associated dominant directions $\left(A^{i}\right)$ of local energy were determined by the basis functions of the constituent filter and its quadrature pair [6].

(3) Coherence Maps: A coherence map is an image showing a local measure of the degree of anisotropy of flow [8]. If the orientations of a texture pattern at any point $\left(x_{i}, y_{i}\right)$ are coherent, then magnitude and phase information are important and should be emphasized. Conversely, if the orientations are not coherent, the magnitude and phase information can be neglected or attenuated. The measure of coherence proposed by Rao and Schunck [8] was obtained by weighting the energy with the normalized projection of energy within a specified window $(\mathcal{W})$ onto the central point $(j, k)$ of each window. This coherence measure incorporates the gradient magnitude and hence places more weight on regions that have higher visual contrast. The combination of coherence and orientation structure was able to extract the more salient features of spiculated lesions. In overview, a schematic diagram of processing Steps 1-3 is shown in Figure 3.

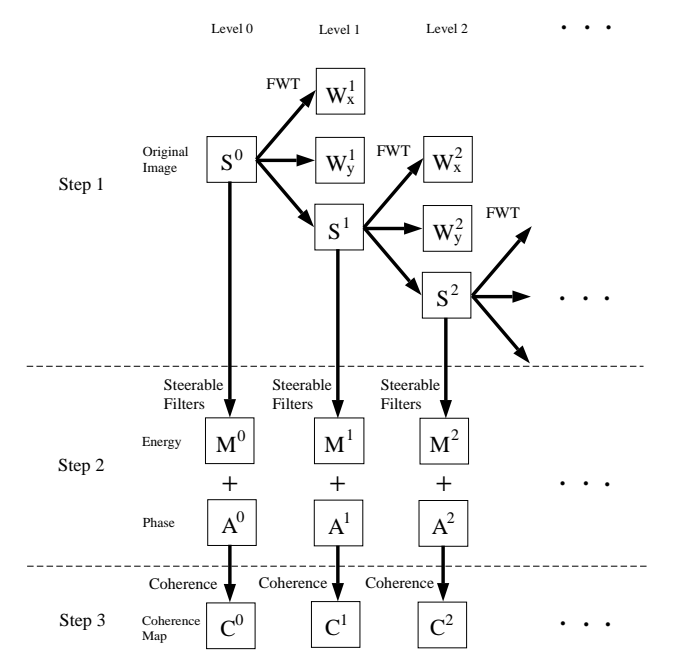

Fig. 3. Overview of processing for Steps 1-3.

(4) Nonlinear Operators: So far, we have precomputed all the information needed in our algorithm. A nonlinear operation was then applied within each 


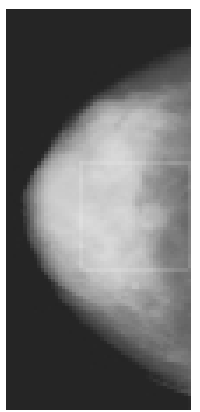

(a)

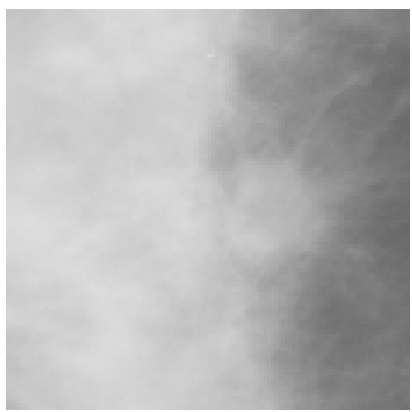

(b)

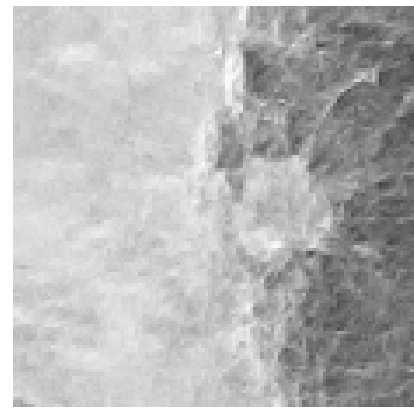

(c)

Fig. 4. Mammogram with a mass: (a) Original mammogram. (b) ROI image. (c) enhanced ROI image.

level to precisely modify transform coefficients. This operation integrates both coherence map and phase information.

A. Modification from Coherence Map: Let $C^{i}(j, k)$ denote the coherence measure of point $(j, k)$ at some level $i$. Modifications of coherence computed in place were obtained by a nonlinear function expressed as

$$
C_{\text {mod }}^{i}(j, k)=\left\{\begin{aligned}
\log \sqrt{C^{i}(j, k)}, & \text { if } C^{i}(j, k)>1, \\
0, & \text { otherwise, }
\end{aligned}\right.
$$

such that a coefficient was emphasized if its coherence measure was large and attenuated if small.

B. Modification from Phase: Phase information is important to distinctly characterize oriented texture. Therefore we did not neglect its contribution in the modification of coefficients. We applied a sinusoidal weighting to the phase information. The final modification was therefore

$$
W_{\text {mod }}^{i}=T^{i-1} \cdot C_{m o d}^{i-1} \cdot A_{m o d}^{i-1} \cdot W^{i}
$$

where $T^{i}$ was a constant at each level and $A_{\text {mod }}^{i}$ was the modified gain from phase. These modified coefficients were then reconstructed, via an inverse fast wavelet transform, to enhance the visualization of possible lesions.

\subsection{Experimental Results}

Our algorithm was then applied to enhance dense mammograms using oriented information. In order to capture distinct directions of subtle features, steerable filters were used. Due to the limit of space we only show one example. A mammogram (mam004lcc) with a mass tumor is shown in Figure 4. The craniocaudal view of the left breast shown in Figure 4(b) shows an irregular spiculated mass in retroglandular fat. Note how the enhanced version shown in Figure 4(c) clearly delineates the margins of the mass. 


\section{Conclusion}

The primary radiographic signs of breast cancer are related to tumor mass, its density, size, shape, borders and calcification content. Extraction of these features and enhancement may assist general radiologists to locate suspicious areas more reliably.

We first provided a more reasonable initialization procedure for wavelet processing. We showed that a continuous scale wavelet detector can provide a more precise matched basis than detectors with a limited number of scales. The importance of employing continuous scale was demonstrated by digital radiographs of a mammography phantom and digitized mammograms. Our investigation showed that the algorithm was able to detect very subtle masses, which were rated to be almost invisible by radiologist specializing in mammography.

Existing and previous multiscale enhancement approaches [3] attempted to enhance an image by detecting edges. Unfortunately, most edge detection algorithms can not distinguish between "authentic" edges and phantom edges. In contrast, we presented an algorithm which relied upon a coherence map and phase information. This resulted in an enhancement naturally close to the original image. Such artifact free processed images provide more familiar visual cues for radiologists.

\section{References}

1. W. T. Freeman and E. H. Adelson. The design and use of steerable filters. IEEE Transactions on Pattern Analysis and Machine Intelligence, 13(9):891-906, 1991.

2. M. Holschneider, R. Kronland-Martinet, J. Morlet, and Ph. Tchamitchian. A realtime algorithm for signal analysis with the help of the wavelet transform. In J. M. Combes, A. Grossmann, and Ph. Tchamitchian, editors, Wavelets: Time-frequency Methods and Phase Space, pages 286-304, Springer-Verlag, Berlin, Germany, 1990.

3. A. F. Laine, S. Schuler, J. Fan, and W. Huda. Mammographic feature enhancement by multiscale analysis. IEEE Transactions on Medical Imaging, 13(4):725-740, 1994.

4. S. Mallat and S. Zhong. Characterization of signals from multiscale edges. IEEE Transactions on Pattern Analysis and Machine Intelligence, 14(7):710-732, 1992.

5. S. D. Marco and J. Weiss. M-band wavepacket-based transient signal detector using a translation-invariant wavelet. Optical Engineering, 33(7):2175-2182, 1994.

6. M. C. Morrone and R. A. Owens. Feature detection from local energy. Pattern Recognition Letters, 6(5):303-313, 1987.

7. P. Perona. Deformable kernels for early vision. IEEE Transactions on Pattern Analysis and Machine Intelligence, 17(5):488-499, 1995.

8. A. R. Rao and B. G. Schunck. Computing oriented texture fields. In Proceedings of the IEEE Computer Society Conference on Computer Vision and Pattern Recognition, pages 61-68, San Diego, CA, 1989.

9. O. Rioul and P. Duhamel. Fast algorithms for discrete and continuous wavelet transforms. IEEE Transactions on Information Theory, 38(2):569-586, 1992.

10. M. Vrhel, C. Lee, and M. Unser. Fast computation of the continuous wavelet transform through oblique projections. In Proceedings of the IEEE International Conference on Acoustics, Speech, and Signal Processing, volume 3, pages 14591462, Atlanta, GA, 1996. 